\title{
Preface
}

\section{Fat Grafting to the Face for Rejuvenation, Contouring, or Regenerative Surgery}

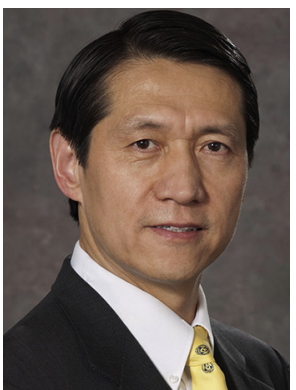

Lee L.Q. Pu, MD, PhD, FACS

Editor

Fat grafting has become one of the most commonly performed procedures in both aesthetic and reconstructive plastic surgery. It started as autologous filler for facial rejuvenation, championed by Dr Sydney Coleman in the mid-1990s, but now it has been used not only for facial rejuvenation but also for facial contouring, another important aspect for facial aesthetic and reconstructive surgery. Until recently, fat grafting has showed its regenerative potential and has also been used to treat some of the difficult clinical problems facing plastic surgeons as an innovative approach called regenerative surgery. As we know more about fat grafting, its mechanisms of how fat grafts survive and their regenerative features, fat grafting, as a relatively noninvasive procedure, may gradually replace many of facial aesthetic and reconstructive procedures in the future. It has become a major armamentarium for plastic surgeons to rejuvenate aged tissues, to contour deficient part of tissues, and to treat certain pathologic conditions of the face.

As a founding member and current board member of International Society of Plastic and Regenerative Surgeons, I believe one of my primary responsibilities is to promote scientific exchange for the art and science of fat grafting. For this reason, I decided to accept another invitation by Elsevier to edit a new issue of Clinics in Plastic Surgery. I have chosen the topic of facial fat grafting because this is the most popular anatomic region that fat grafting is frequently performed for rejuvenation, contouring, or regenerative surgery. I have invited many international contributors who are renowned experts in facial fat grafting not only for facial rejuvenation but also for facial contouring or even for regenerative surgery of the face.

In this issue of 18 articles, the first article, written by $\mathrm{Dr}$ Sydney R. Coleman, serves as an introduction on facial fat grafting that includes his perspectives on the past, present, and future of facial fat grafting. This is followed by an article on the overview of current concepts and techniques of facial fat grafting, a good summary of what we know about facial fat grafting, as well as future development in facial fat grafting. There are a total of 6 articles that focus on facial rejuvenation, starting with more established technique and followed by more contemporary and advanced techniques for facial rejuvenation. In addition, facial fat grafting with nanofat grafts, cryopreserved fat grafts, and stromal vascular fraction gel are also included as an individual article. Since facial fat grafting has been commonly used in Asia for facial contouring, a total of 4 articles are included that focus on contouring for temporal region and midface, nose and chin, unilateral face, and even for panfacial contouring. In this issue, an additional 5 articles are included as an innovative approach of regenerative surgery to the face. These articles include fat grafting for treatment of facial burn and burn scars, for treatment of facial scars, for treatment of secondary facial deformity, and last, for treatment of facial scleroderma. The last article focuses on 
prevention and management of serious complications after facial fat grafting. This article includes unique experiences of our Chinese colleagues, who have been known for the management of serious complications after facial fat grafting. Therefore, this issue represents the most updated information on facial fat grafting for rejuvenation, facial contouring, or regenerative surgery.

As guest editor, I sincerely hope that you will enjoy reading this special issue of Clinics in Plastic Surgery. It represents a true team effort from many world-renowned experts from the United States, Greater China, Italy, Belgium, Japan, Italy, Brazil, Germany, and France. I would like to express my heartfelt gratitude to all contributors for their expertise, dedication, and responsibility to produce such a world-class issue of plastic surgery. It is certainly my privilege to work with these respected authors in this exciting field of plastic surgery. I would also like to express my special appreciation to the publication team of Elsevier, who has put this remarkable issue together with the highest possible standard.

Lee L.Q. Pu, MD, PhD, FACS

Division of Plastic Surgery University of California Davis 2335 Stockton Boulevard, Suite 6008 Sacramento, CA 95817, USA 